\title{
Effect of stress-jump condition on electrophoretic behavior of a spherical dispersion of soft particles
}

\author{
Eric Lee, Wen-Li Cheng, Jyh-Ping Hsu* \\ Department of Chemical Engineering, National Taiwan University, Taipei 10617, Taiwan \\ Received 22 August 2005; accepted 8 September 2005 \\ Available online 2 November 2005
}

\begin{abstract}
The electrophoresis of a concentrated dispersion of soft particles, where a particle comprises a rigid core and an ion-penetrable membrane layer, is modeled theoretically, taking the effect of double-layer polarization into account. In particular, the influence of a stress-jump condition of the flow field at the membrane layer-liquid interface on the electrophoretic mobility of a particle is investigated. The type of particles considered mimic biocolloids, such as cells and microorganisms, and inorganic colloids covered by an artificial polymer layer such as surfactant molecules. A unit cell model is adopted to simulate the present spherical dispersion, and the governing equations and the associated boundary conditions are solved by a pseudo-spectral method based on Chebyshev polynomials. We show that while the stress-jump condition, characterized by a stress-jump coefficient, can have a significant influence on the mobility of a particle, the associated flow field is not influenced appreciably. Also, the influence of the stress-jump condition on the mobility of a particle depends largely on the nature of the membrane layer, characterized by its friction coefficient.
\end{abstract}

(C) 2005 Elsevier Inc. All rights reserved.

Keywords: Electrophoresis; Concentrated dispersion; Soft particles; Unit cell model; Double-layer polarization; Stress-jump condition

\section{Introduction}

Recently, the electrophoretic behavior of soft particles, namely, particles comprising a rigid core and a porous layer, has drawn the attention of researchers in various fields. Biocolloids such as cells and microorganisms and inorganic particles covered by a surfactant or polymer layer are typical examples of particles of this type. Apparently, to predict the electrophoretic behavior of such particles, the classic theory of electrophoresis, which is valid for rigid particles, needs to be modified to take into account the presence of the membrane layer. Although relevant analyses are ample in the literature (e.g., [1-9]), the influence of the specific behavior of the flow field across the membrane layer-liquid interface on the electrophoretic behavior of a particle has not been reported.

The boundary condition for the flow field across a porous material-liquid interface has drawn the attention of many re-

\footnotetext{
* Corresponding author. Fax: +8862 23623040.

E-mail address: jphsu@ntu.edu.tw (J.-P. Hsu).
}

searchers. Based on experimental observation, Beavers and Joseph [10] pointed out that the fluid velocity at the interface of a porous medium and liquid phase might be of a slip nature. An empirical expression, which relates the slip velocity and the exterior flow, was proposed, and it was capable of explaining the experimental data gathered. The boundary condition of Beavers and Joseph was also used by Vafai and co-workers $[11,12]$ in a heat transfer problem. The Darcy equation was used as the governing equation for the flow field in the porous region of Beavers and Joseph [10]. Because the order of fluid velocity in this equation is different from that of the Stokes equation in the free liquid region, solving the governing equations for the whole system becomes overdetermined when the condition that the shear stress on the porous mediumliquid interface is continuous is imposed. Although this difficulty can be circumvented by replacing the Darcy equation with the Brinkman equation, the treatment of the tangential stress at the interface is still unsatisfactory, and it is often assumed that this stress is continuous. Based on the idea of volume averaging, Ochoa-Tapia and Whitaker [13] proposed that while the fluid velocity is continuous across the porous medium-liquid 
interface, the corresponding shear stress is not. An extra stress term, called stress jump, was added in the equation of motion in their treatment to associate the Brinkman equation and Stokes equation. The stress jump is characterized by a stress-jump coefficient, a real number of magnitude on the order of unity. The treatment of Ochoa-Tapia and Whitaker is not only physically more precise than that of Beavers and Joseph [10] but also more persuasive in the explanation of their experimental observations [14]. Kuznetsov [15] adopted the stress-jump condition of Ochoa-Tapia and Whitaker [13] to extend the analysis of Vafai and co-workers [11,12] and concluded that the fluid velocity at a porous material-liquid interface varies with the stress-jump coefficient. This implies that the latter is not just an adjustable parameter, but plays an important role in describing the flow field associated with porous material. In a study of twodimensional viscous flow, Raja Sekhar and Sano [16] adopted the stress-jump condition of Ochoa-Tapia and Whitaker [13], and found that the value of the stress-jump coefficient ranged from about -0.9 to 0.9 . Bhattacharyya and Raja Sekhar [17] investigated the relation between the stress-jump condition and various types of viscous flow by considering a spherical particle comprising a rigid core and a porous shell and concluded that taking the stress-jump condition into account was necessary.

In this study the influence of the discontinuity of the flow field across the porous material-liquid interface on the electrophoretic behavior of a spherical dispersion of porous colloidal particles is examined. The stress-jump condition of Ochoa-Tapia and Whitaker [13] is adopted for this purpose, and the unit cell model of Kuwabara [18] is chosen to simulate the dispersion. The equations governing the problem under consideration and the associated boundary conditions are solved numerically by a pseudo-spectral method based on Chebyshev polynomials [9].

\section{Theory}

We consider the electrophoresis of a spherical dispersion of soft particles where a particle comprises a rigid core of radius $a$ and a membrane layer of thickness $d$. Let $b=a+d$. The liquid phase contains a $z_{1}: z_{2}$ electrolyte, $z_{1}$ and $z_{2}$ being respectively the valences of cations and ions with $z_{2}=-\alpha z_{1}$. As illustrated in Fig. 1, the unit cell model of Kuwabara [18] is adopted, where a dispersion is simulated by a representative particle that is surrounded by a spherical liquid shell of radius $c$. The volume fraction of particles is measured by the ratio $H=(b / c)^{3}$. The spherical coordinates $(r, \theta, \varphi)$ are used with their origin located at the center of the representative particle and the symmetric axis, $\theta=0$, parallel to the applied electric field $\mathbf{E} ; E z$ is its $z$-component. Let $\mathbf{U}$ be the electrophoretic velocity of the particle.

For the present problem the electric field, the concentration field, and the flow field need to be solved simultaneously. The governing equation for these fields can be expressed as

$$
\begin{aligned}
& \nabla^{2} \phi=-\frac{\rho+\rho_{\text {fix }}}{\varepsilon}, \quad a<r<b, \\
& \nabla^{2} \phi=-\frac{\rho}{\varepsilon}, \quad b<r<c,
\end{aligned}
$$

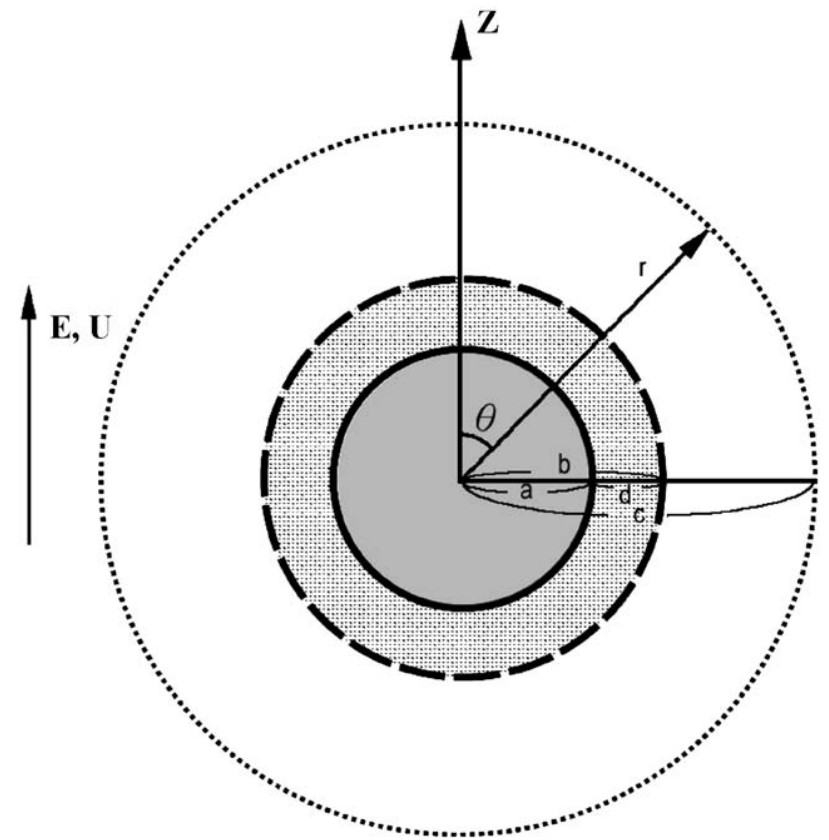

Fig. 1. Kuwabara's unit cell model [18], where a spherical dispersion is simulated by a representative particle comprising a rigid core of radius $a$ and a membrane layer of thickness $d, b=a+d$, and a spherical liquid shell of radius $c .(r, \theta, \varphi)$ is the spherical coordinates with their origin located at the center of the particle and the symmetric axis, $\theta=0$, is parallel to the applied electric field $\mathbf{E}$ and the electrophoretic velocity of the particle $\mathbf{U}$.

$-D_{j}\left[\nabla^{2} n_{j}+\frac{z_{j} e}{k T}\left(\nabla n_{j} \cdot \nabla \phi+n_{j} \nabla^{2} \phi\right)\right]+\nabla n_{j} \cdot \mathbf{v}=0$,

$\nabla \cdot \mathbf{v}=0$

$-\nabla p+\eta \nabla^{2} \mathbf{v}-\rho \nabla \phi-\gamma \mathbf{v}=0, \quad a<r<b$,

$-\nabla p+\eta \nabla^{2} \mathbf{v}-\rho \nabla \phi=0, \quad b<r<c$.

In these expressions, $\phi$ is the electric potential, $\nabla$ is the gradient operator, $\nabla^{2}$ is the Laplacian, $\rho=\sum_{j} z_{j} n_{j} e$ is the space charge density, $n_{j}, z_{j}$, and $D_{j}$ are respectively the number density, the valence, and the diffusivity of ionic species $j, k$, and $T$ are respectively the Boltzmann constant and the absolute temperature, $\mathbf{v}$ is the liquid velocity, $e$ and $\varepsilon$ are respectively the elementary charge and the dielectric constant, $\rho_{\text {fix }}$ is the fixed charge density in the membrane layer, $p$ is the pressure, $\eta$ and $\rho$ are respectively the viscosity and the space charge density, and $\gamma$ is the friction coefficient of the membrane layer. Here, we assume that the liquid phase is an incompressible Newtonian fluid and both $\rho_{\mathrm{fix}}$ and $\varepsilon$ are position-independent. Note that assuming a position-dependent dielectric constant in the membrane layer yields a more complicated description for the electric field. Since it is expected that the qualitative behavior of the system under consideration remains similar, a constant dielectric constant is assumed in our analysis.

We assume that the physical properties of both the membrane layer and the liquid phase remain at constant values, the liquid phase is an incompressible Newtonian fluid, and the surface of the rigid core of a particle remains at a constant electrical potential. Also, the applied electric field is relatively weak compared with that due to the presence of a particle. For easier mathematical treatment, $\phi$ is decomposed into the electrical 
potential in the absence of $\mathbf{E}$, or the equilibrium electrical potential, $\phi_{\mathrm{e}}$, and that when $\mathbf{E}$ is applied, $\delta \phi$ [19]. The effect of double-layer polarization is taken into account by letting [19]

$n_{j}=n_{j_{0}} \exp \left[-\frac{z_{j} e}{k T}\left(\phi_{\mathrm{e}}+\delta \phi+g_{j}\right)\right]$,

where $n_{j_{0}}$ is the bulk concentration of ionic species $j$, the term $g_{j}$ explains the distortion of the ionic cloud surrounding a particle arising from the flow field. Substituting Eq. (7) into Eqs. (1)-(3) and using $\phi=\phi_{\mathrm{e}}+\delta \phi$, we obtain

$$
\begin{aligned}
\nabla^{2} \phi_{\mathrm{e}}= & -\sum_{j=1}^{2} \frac{z_{j} e n_{j_{0}}}{\varepsilon} \exp \left(-\frac{z_{j} e \phi_{\mathrm{e}}}{k T}\right)-\frac{\rho_{\mathrm{fix}}}{\varepsilon}, \quad a<r<b, \\
\nabla^{2} \phi_{\mathrm{e}}= & -\sum_{j=1}^{2} \frac{z_{j} e n_{j_{0}}}{\varepsilon} \exp \left(-\frac{z_{j} e \phi_{\mathrm{e}}}{k T}\right), \quad b<r<c, \\
\nabla^{2} \delta \phi= & \nabla^{2} \phi-\nabla^{2} \phi_{\mathrm{e}} \\
= & -\sum_{j=1}^{2} \frac{z_{j} e n_{j_{0}}}{\varepsilon}\left(\exp \left(-\frac{z_{j} e\left(\phi_{\mathrm{e}}+\delta \phi+g_{j}\right)}{k T}\right)\right. \\
& \left.-\exp \left(-\frac{z_{j} e \phi_{\mathrm{e}}}{k T}\right)\right), \\
\nabla^{2} g_{j}- & \frac{z_{j} e}{k T} \nabla \phi_{\mathrm{e}} \cdot \nabla g_{j}=\frac{1}{D_{j}} \mathbf{v} \cdot \nabla \phi+\frac{1}{D_{j}} \mathbf{v} \cdot \nabla g_{j} \\
& +\frac{z_{j} e}{k T} \nabla \delta \phi \cdot \nabla g_{j} \\
& +\frac{z_{j} e}{k T} \nabla g_{j} \cdot \nabla g_{j} .
\end{aligned}
$$

In terms of the stream function $\psi$, it can be shown that Eqs. (5) and (6) lead to

$$
\begin{aligned}
& E^{4} \psi-\gamma E^{2} \psi=-\frac{\sin \theta}{\eta} \nabla \times\left[\rho \nabla\left(\phi_{\mathrm{e}}+\delta \phi\right)\right], \\
& \quad a<r<b, \\
& E^{4} \psi=-\frac{\sin \theta}{\eta} \nabla \times\left[\rho \nabla\left(\phi_{\mathrm{e}}+\delta \phi\right)\right], \quad b<r<c,
\end{aligned}
$$

where $E^{4}=E^{2} E^{2}$, and in spherical coordinates $E^{2}$ is

$E^{2} \equiv \frac{\partial}{\partial r^{2}}+\frac{\sin \theta}{r^{2}} \frac{\partial}{\partial \theta}\left(\frac{1}{\sin \theta} \frac{\partial}{\partial \theta}\right)$.

In terms of $\psi$, the $r$ - and the $\theta$-components of $\mathbf{v}, v_{r}$ and $v_{\theta}$, can be expressed respectively as $v_{r}=-\frac{1}{r^{2} \sin \theta} \frac{\partial \dot{\psi}}{\partial \theta}$ and $v_{\theta}=$ $\frac{1}{r \sin \theta} \frac{\partial \psi}{\partial r}$.

\section{Boundary conditions}

We assume that the electric potential on the surface of the rigid core of a particle $(r=a)$ is remained at the constant value of $\zeta$, the surface is impenetrable to ions and is nonslip. Suppose that the electric potential, the electric field, the concentration, and the flux of ions are continuous on the surface of the soft layer of a particle $(r=b)$. The electroneutrality of the system under consideration requires that there is no net current across the surface of a cell $(r=c)$. Also, the concentrations of ions arrive at the corresponding bulk values on this surface. According to Levine and Neale [20], the electric field on that surface is that which arises from the applied electric field. The boundary condition of Ochoa-Tapia and Whitaker [13] on the surface of the soft layer of a particle $(r=b)$ is adopted; that is, the velocity of fluid is continuous, but a correction factor $\beta$ is introduced for the corresponding tangential stress. Also, we assume that the vorticity on cell surface vanishes and there is no net flow between adjacent cells. Based on these conditions, the boundary conditions associated with the present problem are

$$
\begin{aligned}
& \phi_{\mathrm{e}}=\zeta, \quad r=a, \\
& \left.\phi_{\mathrm{e}}\right|_{r=b^{-}}=\left.\phi_{\mathrm{e}}\right|_{r=b^{+}}, \quad r=b, \\
& \left.\frac{\partial \phi_{\mathrm{e}}}{\partial r}\right|_{r=b^{-}}=\left.\frac{\partial \phi_{\mathrm{e}}}{\partial r}\right|_{r=b^{+}}, \quad r=b, \\
& \frac{\partial \phi_{\mathrm{e}}}{\partial r}=0, \quad r=c, \\
& \frac{\partial \delta \phi}{\partial r}=0, \quad r=a, \\
& \left.\delta \phi\right|_{r=b^{-}}=\left.\delta \phi\right|_{r=b^{+}}, \quad r=b, \\
& \left.\frac{\partial \delta \phi}{\partial r}\right|_{r=b^{-}}=\left.\frac{\partial \delta \phi}{\partial r}\right|_{r=b^{+}}, \quad r=b, \\
& \frac{\partial \delta \phi}{\partial r}=-E_{z} \cos \theta, \quad r=c, \\
& \left(\mathbf{f}_{j}-n_{j} \mathbf{v}\right) \cdot \mathbf{r}=0, \quad r=a, \\
& \left.n_{j}\right|_{r=b^{-}}=\left.n_{j}\right|_{r=b^{+}}, \quad r=b, \\
& \left.\mathbf{f}_{j}\right|_{r=b^{-}}=\left.\mathbf{f}_{j}\right|_{r=b^{+}}, \quad r=b, \\
& n_{j}=n_{j_{0}}, \quad r=c \text {, } \\
& v_{r}=U \cos \theta \quad \text { and } \quad v_{\theta}=-U \sin \theta, \quad r=a \text {, } \\
& \left.v_{r}\right|_{r=b^{-}}=\left.v_{r}\right|_{r=b^{+}},\left.\quad v_{\theta}\right|_{r=b^{-}}=\left.v_{\theta}\right|_{r=b^{+}}, \quad r=b, \\
& \left.\sigma_{r \theta}^{H}\right|_{r=b^{-}}+\beta \sqrt{\eta \gamma} v_{\theta}=\left.\sigma_{r \theta}^{H}\right|_{r=b^{+}}, \quad r=b, \\
& \left.\sigma_{r r}^{T}\right|_{r=b^{-}}=\left.\sigma_{r r}^{T}\right|_{r=b^{+}} \quad \text { and }\left.\quad \sigma_{r r}^{E}\right|_{r=b^{-}}=\left.\sigma_{r r}^{E}\right|_{r=b^{+}} \text {, } \\
& r=b, \\
& \nabla \times \mathbf{v}=0 \quad \text { and } \quad v_{r}=0, \quad r=c .
\end{aligned}
$$

In these expressions, $\mathbf{f}_{j}$ is the concentration flux of ionic species $j, n$ is the unit normal vector, $\mathbf{r}$ is the unit vector in the $r$-direction, $\sigma_{r \theta}^{H}$ is the $r \theta$-component of the hydrodynamic stress $\sigma^{H}, \sigma_{r r}^{E}$ is the $r r$-component of the Maxwell stress $\sigma^{E}$, and $\sigma_{r r}^{T}$ is the $r r$-component of the total stress $\sigma^{T}=\sigma^{H}+\sigma^{E}$.

The governing equations are solved subject to the associated boundary conditions by a pseudo-spectral method proposed by Lee et al. [9] and the electrophoretic mobility of a particle is calculated based on the approach of O'Brien and White [19].

\section{Results and discussion}

For convenience, the following dimensionless symbols are used in subsequent discussions: $\mu_{\mathrm{m}}^{*}=U^{*} / E_{z}^{*}=\eta U / \varepsilon \zeta E_{z}$ is the scaled mobility, $(\lambda a)^{2}=\left(\gamma a^{2} / \eta\right)$ is a measure for the significance of the drag of membrane layer, $\psi^{*}=\psi / U_{E} a$ is the scaled stream function where $U_{E}=\varepsilon \zeta^{2} / \eta a$ is the electrophoretic velocity based on Smoluchowski's formula when an electric field $(\zeta / a)$ is applied. $E_{Z}^{*}=E_{Z} /(\zeta / a)$ is the scaled 


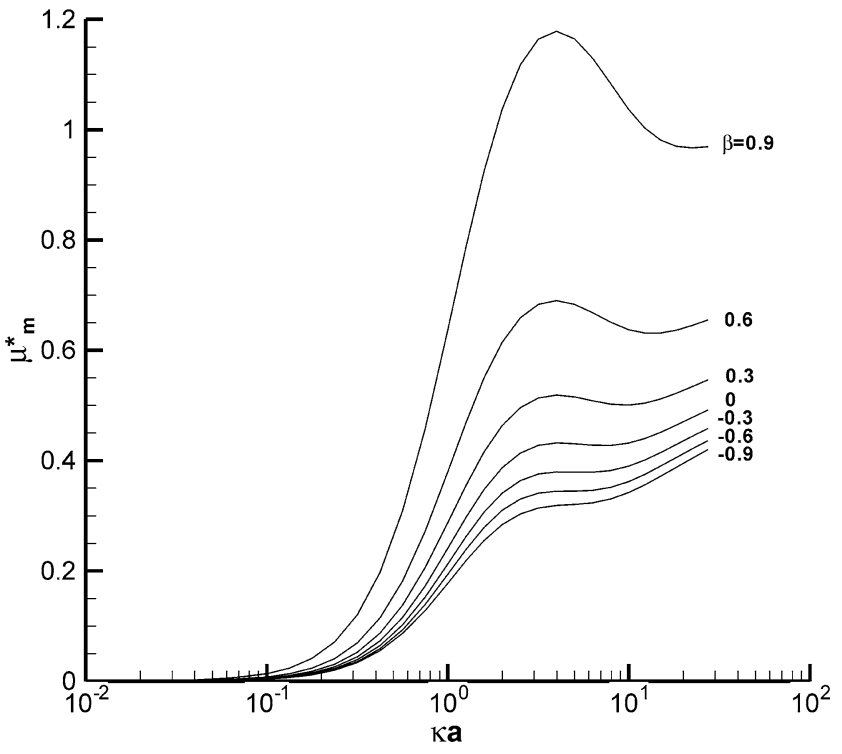

Fig. 2. Variation of scaled mobility as a function of $\kappa a$ at various $\beta$ for the case when $Q_{\text {fix }}=0$ and $\lambda a=5$. Key: $\phi_{r}=1.0, H=0.421875, \mathrm{Pe}_{1}=\mathrm{Pe}_{2}=0.01$, and $d / a=0.5$.

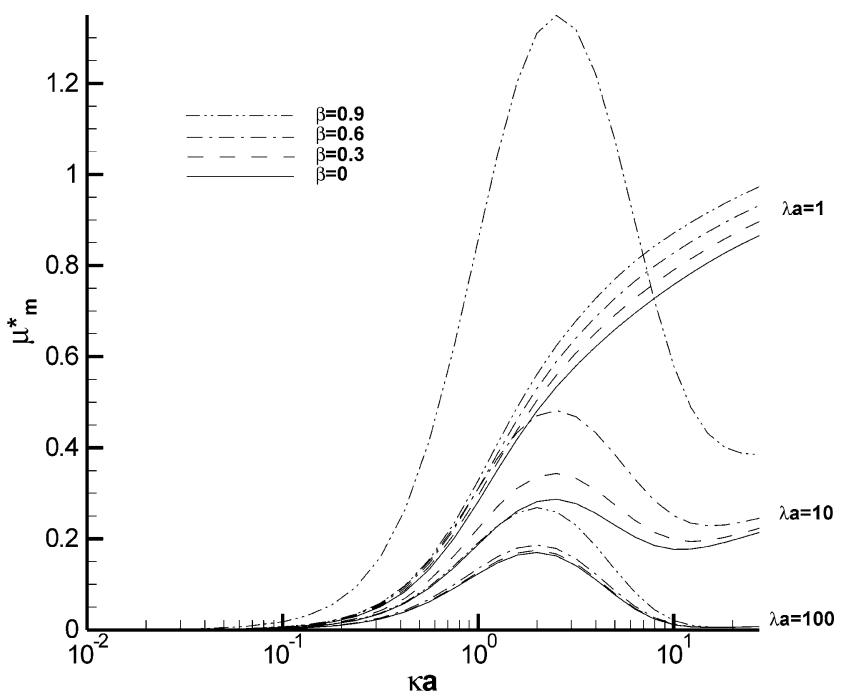

Fig. 3. Variation of scaled mobility as a function of $\kappa a$ at various $\beta$ and $\lambda a$ for the case when $Q_{\text {fix }}=0$. Key: same as in Fig. 2 .

applied electric field in the $z$-direction, $\phi_{\mathrm{e}}^{*}=\phi_{\mathrm{e}} / \zeta$ is the scaled equilibrium electrical potential, $\kappa a$ is the scaled double-layer thickness, where $\kappa^{-1}=\left(\varepsilon k T / \sum n_{j_{0}}\left(e z_{j}\right)^{2}\right)^{1 / 2}$ is the Debye length, and $\phi_{r}=z_{1} e \zeta / k T$ is the scaled surface potential. $Q_{\mathrm{fix}}=\left(\rho_{\mathrm{fix}} a^{2} / \varepsilon \zeta\right)$ is the scaled amount of fixed charge in the membrane layer of a particle, and $\mathrm{Pe}_{i}=U_{E} a / D_{i}$ is the electric Peclet number for ionic species $i$.

Fig. 2 shows that if both $\lambda a$ and $\kappa a$ are fixed, the mobility of a particle correlates positively with $\beta$. This is expected because varying the magnitude of $\beta$ has the effect of changing the flow field in the membrane layer of a particle, and its velocity is influenced accordingly. Note that if $\lambda a$ is either very large or very small, the mobility of a particle becomes insensitive to the variation of $\beta$, as can be seen in Fig. 3. This is because if $\lambda a \rightarrow 0$, the membrane layer is absent as far as the flow field

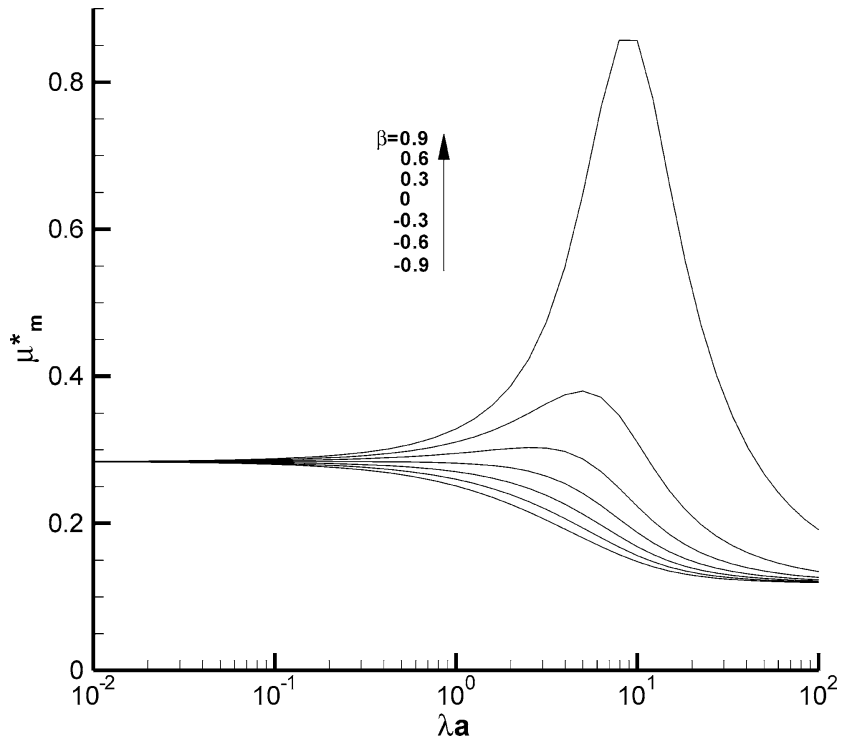

Fig. 4. Variation of scaled mobility as a function of $\lambda a$ at various $\beta$ for the case when $Q_{\text {fix }}=0$ and $\kappa a=1$. Key: same as in Fig. 2.

is concerned, and the behavior of the system under consideration is similar to that of a spherical dispersion of rigid particles of radius $a$. Similarly, the behavior of the system under consideration is similar to that of a spherical dispersion of rigid particles of radius $b(=a+d)$ as $\lambda a \rightarrow \infty$. The influence of $\beta$ in these two extreme cases is negligible. Fig. 3 also reveals that if $\lambda a$ is small, the scaled mobility of a particle $\mu_{\mathrm{m}}^{*}$ increases monotonically with the increase in $\kappa a$ for the range of $\kappa a$ considered; if $\lambda a$ is large, $\mu_{\mathrm{m}}^{*}$ has a local maximum as $\kappa a$ varies, and it has both a local maximum and a local minimum if $\lambda a$ takes a medium value. This implies that the nature of the membrane layer of a particle can have a profound influence on its electrophoretic behavior. It is interesting to note that for a fixed value of $\lambda a$, the value of $\kappa a$ at which the local maximum of $\mu_{\mathrm{m}}^{*}$ occurs is the same, and ranges from 2 to 3 ; that is, the double layer surrounding a particle is enclosed in its membrane layer. This implies that $\beta$ is unable to influence the phenomenon of double-layer polarization observed in Lee et al. [9]; that is, $\beta$ and $\kappa a$ are independent of each other.

Fig. 4 reveals that the scaled mobility of a particle $\mu_{\mathrm{m}}^{*}$ for $\beta \leqslant 0$ is less sensitive to the variation of $\lambda a$ than that for $\beta>0$. In the latter, $\mu_{\mathrm{m}}^{*}$ has a local maximum, and the larger the $\beta$ the more sensitive is $\mu_{\mathrm{m}}^{*}$ to the variation of $\lambda a$. Note that if $\beta$ depends only on the nature of the membrane layer-liquid interface and the fluid outside a particle, then the value of $\lambda a$ at which the local maximum of $\mu_{\mathrm{m}}^{*}$ occurs, should not vary with $\beta$. Therefore, we conclude that $\beta$ and $\lambda a$ are correlated.

Fig. 5 illustrates the variation of the scaled mobility of a particle $\mu_{\mathrm{m}}^{*}$ as a function of $\kappa a$ at various values of $\lambda a$ when the membrane layer of a particle carries negative fixed charge, and Fig. 6 shows that at various values of $H$. As can be seen in Fig. 5, a positive value of $\beta$ leads to an increase in $\left|\mu_{\mathrm{m}}^{*}\right|$, and this effect is pronounced when $\lambda a$ takes a medium value. This is consistent with the results shown in Fig. 3 where the membrane layer of a particle is free of fixed charge. According to Fig. 6, the influences of $\beta$ on the value of $\mu_{\mathrm{m}}^{*}$ at various concentrations 


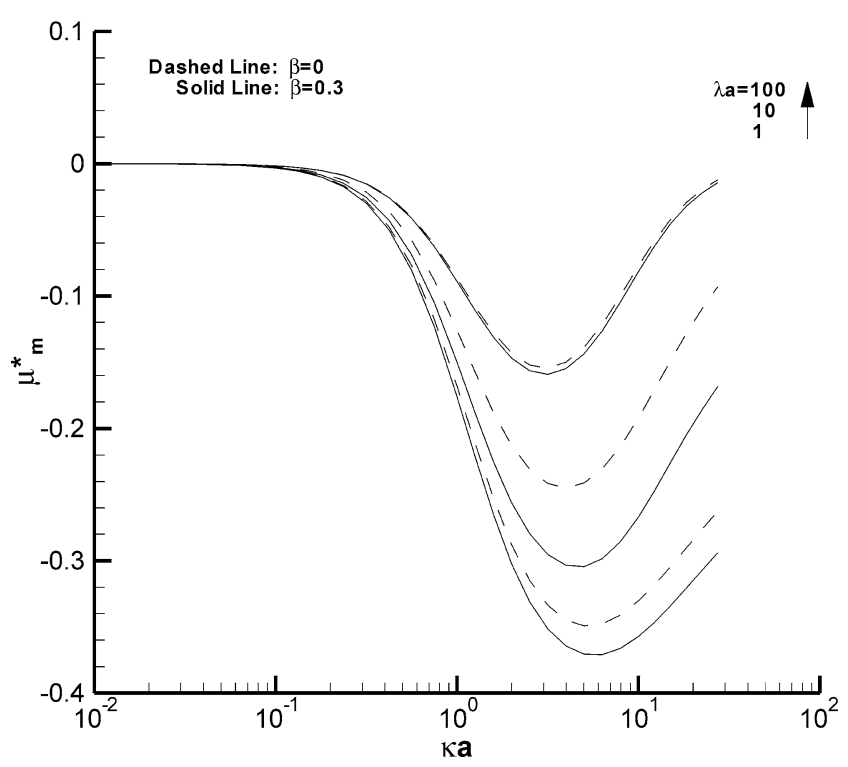

Fig. 5. Variation of scaled mobility as a function of $\kappa a$ at various $\beta$ and $\lambda a$ for the case when $Q_{\text {fix }}=-10$. Key: same as in Fig. 2.

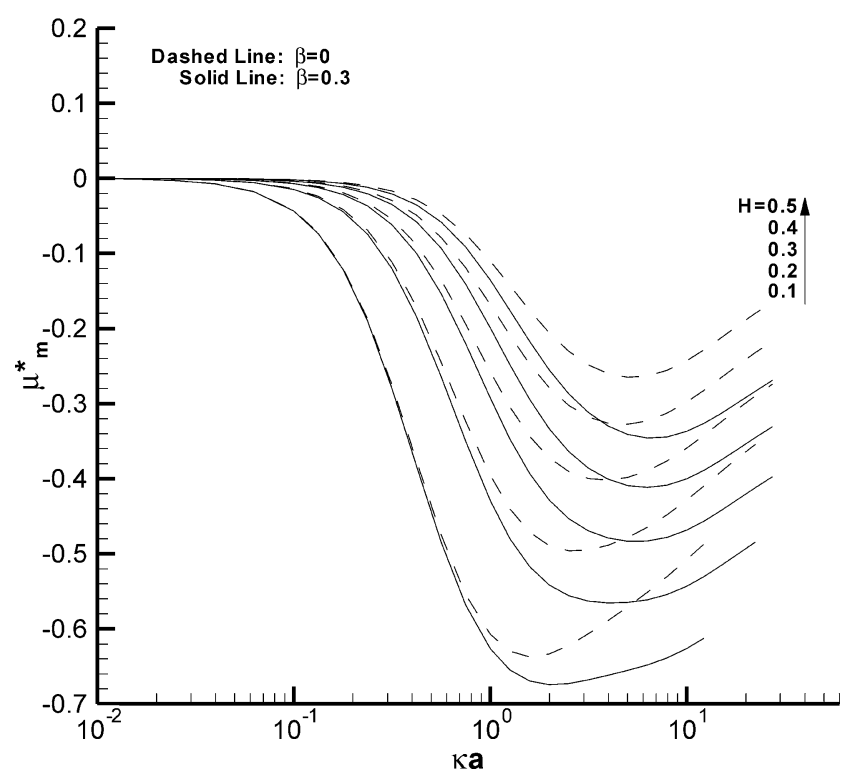

Fig. 6. Variation of scaled mobility as a function of $\kappa a$ at various $\beta$ and $H$ for the case when $Q_{\mathrm{fix}}=-10, \lambda a=5, \phi_{r}=1.0$, and $d / a=0.5$.

of particles are on the same level. This is because both the value of $\lambda a$ and the thickness of the membrane layer of a particle are fixed. Therefore we conclude that $\beta$ is independent of $H$.

Figs. 7-9 show some typical flow fields of the problem under consideration. These figures reveal that the qualitative nature of the contours of stream lines is not influenced appreciably by the introduction of $\beta$, especially when $\lambda a=1$, which is expected since the presence of the membrane layer can be neglected in this case. However, as $\lambda a$ gets large, the contours of the stream lines in the region between the outer surface of the membrane layer and cell boundary becomes more uniform. Figs. 7-9 suggest that the introduction of a positive $\beta$ has the effect of raising the gradient of the stream line near the surface of a particle. Since $v_{\theta}$ is proportional to the gradient of stream

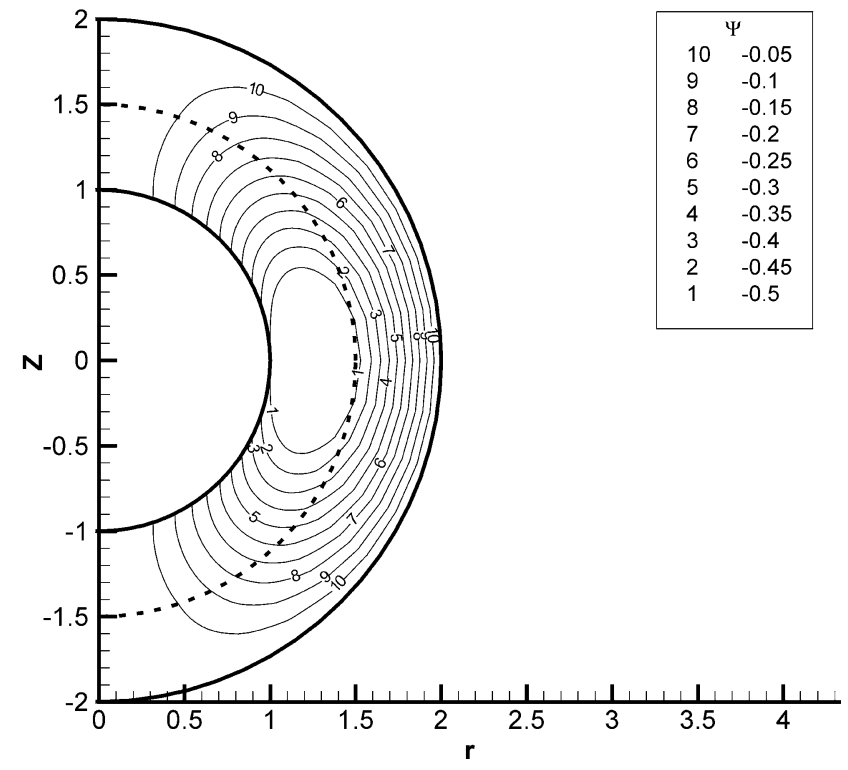

(a)

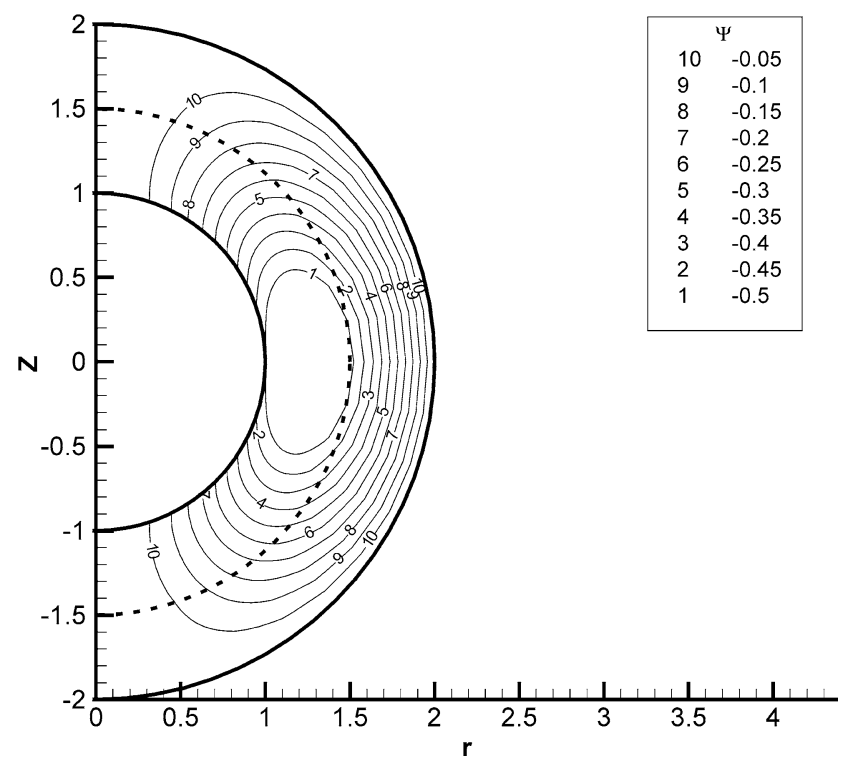

(b)

Fig. 7. The stream function profile for the case when $Q_{\text {fix }}=-10, \kappa a=1$, and $\lambda a=1$. (a) $\beta=0$, (b) $\beta=0.9$. Key: same as in Fig. 2 .

line, this means that the mobility of a particle increases accordingly.

\section{Conclusions}

The electrophoresis of a concentrated dispersion of soft particles, where a particle comprises a rigid core and an ionpenetrable membrane layer, is analyzed taking account of the effect of a stress-jump condition of the flow filed on the membrane layer-liquid interface. We show that this condition, characterized by a stress-jump coefficient, can have a significant influence on the mobility of a particle. While that influence depends largely on the nature of a membrane layer, characterized by its friction coefficient, it is independent of the associated electrical field characterized by the double layer surrounding a 


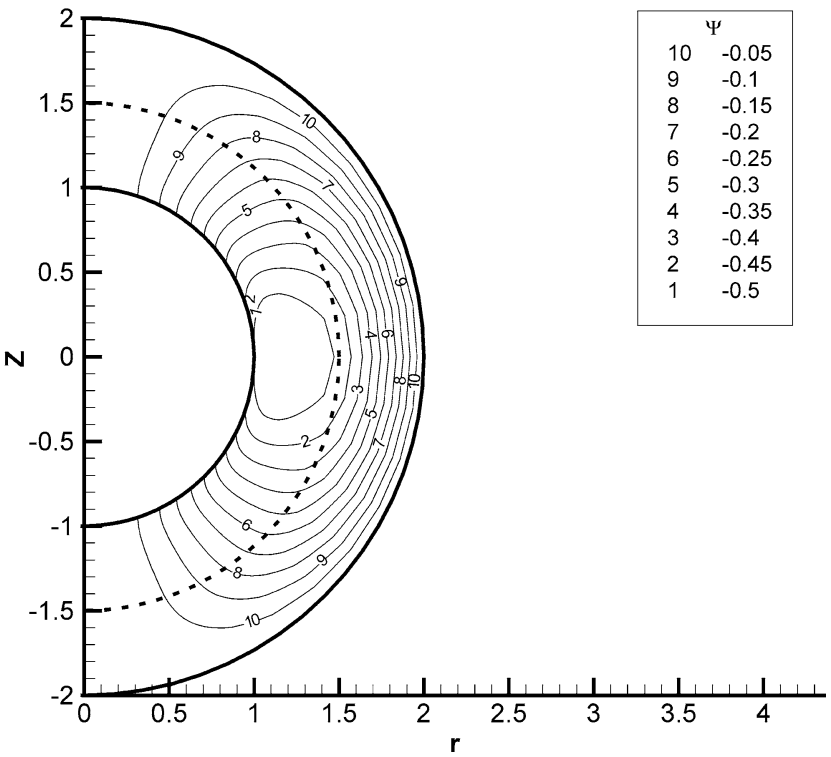

(a)

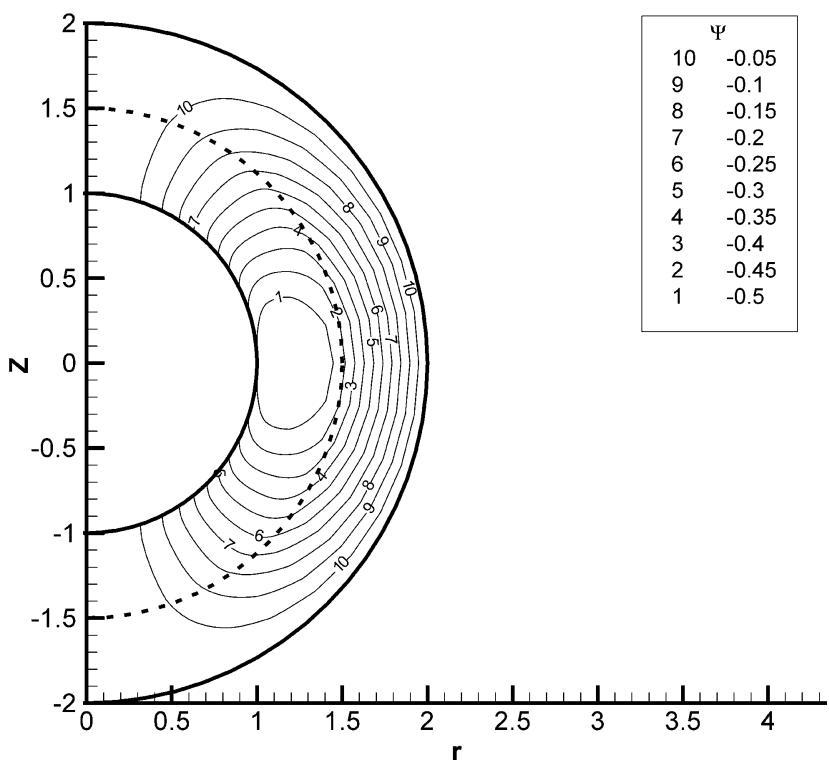

(b)

Fig. 8. The stream function profile for the case when $Q_{\text {fix }}=-10, \kappa a=1$, and $\lambda a=10$. (a) $\beta=0$, (b) $\beta=0.9$. Key: same as in Fig. 2 .

particle. Since the stress-jump coefficient is correlated with the friction coefficient, the influence of the membrane layer of a particle on its mobility is mainly affected by the latter, which is material-dependent.

\section{Acknowledgment}

This work is supported by the National Science Council of the Republic of China.

\section{References}

[1] E. Donath, V. Pastushenko, Bioelectrochem. Bioenerg. 6 (1979) 543.

[2] R.W. Wunderlich, J. Colloid Interface Sci. 88 (1982) 385.

[3] S. Levine, M. Levine, K.A. Sharp, D.E. Brooks, Biophys. J. 42 (1983) 127.

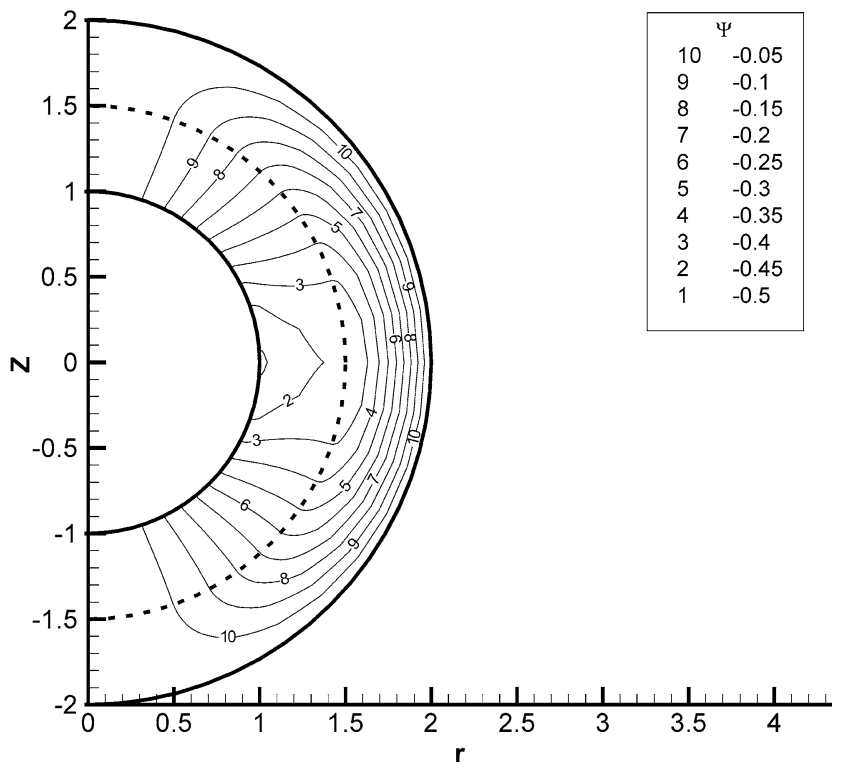

(a)

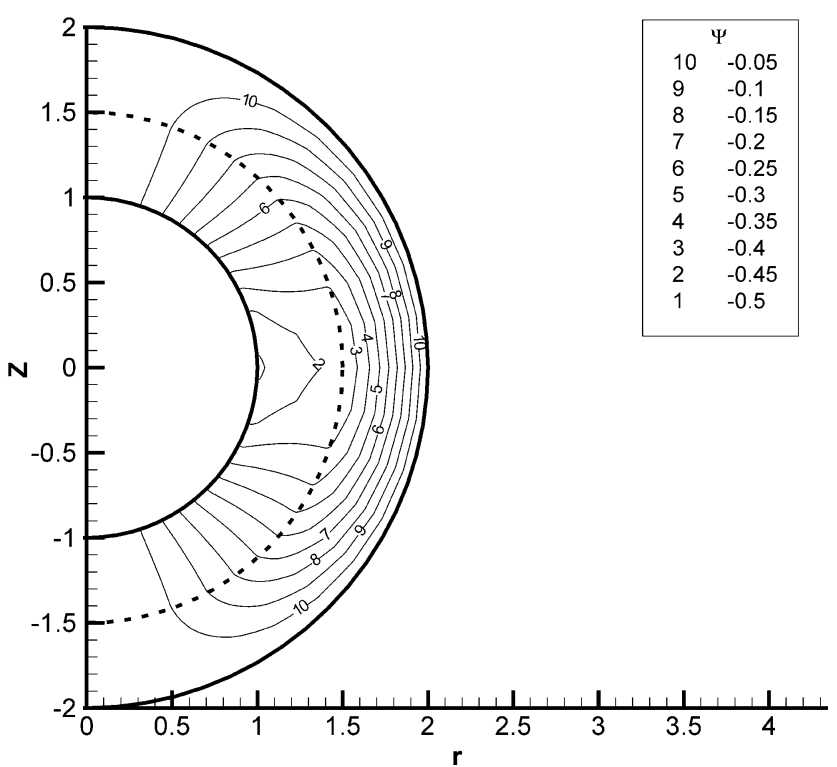

(b)

Fig. 9. The stream function profile for the case when $Q_{\text {fix }}=-10, \kappa a=1$, and $\lambda a=100$. (a) $\beta=0$, (b) $\beta=0.9$. Key: same as in Fig. 2 .

[4] K.A. Sharp, D.E. Brooks, Biophys. J. 47 (1985) 563.

[5] H. Ohshima, J. Colloid Interface Sci. 163 (1994) 474.

[6] H. Ohshima, J. Colloid Interface Sci. 228 (2000) 190.

[7] D.A. Saville, J. Colloid Interface Sci. 222 (2000) 137.

[8] D.A. Saville, J. Colloid Interface Sci. 258 (2003) 56.

[9] E. Lee, K.T. Chou, J.P. Hsu, J. Colloid Interface Sci. 280 (2004) 518.

[10] G.S. Beavers, D.D. Joseph, J. Fluid Mech. 30 (1967) 197.

[11] K. Vafai, R. Thiyagaraja, Int. J. Heat Mass Transfer 30 (1987) 1391.

[12] K. Vafai, S.J. Kim, Int. J. Heat Fluid Flow 11 (1990) 254.

[13] J.A. Ochoa-Tapia, S. Whitaker, Int. J. Heat Mass Transfer 38 (1995) 2635.

[14] J.A. Ochoa-Tapia, S. Whitaker, Int. J. Heat Mass Transfer 38 (1995) 2647.

[15] A.V. Kuznetsov, Int. J. Heat Mass Transfer 24 (1997) 401.

[16] G.P. Raja Sekhar, O. Sano, Phys. Fluids 15 (2003) 554.

[17] A. Bhattacharyya, G.P. Raja Sekhar, Chem. Eng. Sci. 59 (2004) 4481.

[18] S. Kuwabara, J. Phys. Soc. Jpn. 14 (1959) 527.

[19] R.W. O'Brien, L.R. White, J. Chem. Soc. Faraday Trans. 2 (74) (1978) 1607.

[20] S. Levine, G.H. Neale, J. Colloid Interface Sci. 47 (1974) 520. 